\title{
Políticas de la catástrofe.
}

\section{Nuevo paradigmas entre excepción, gobierno, riesgo y soberanía}

\author{
Giulio Nicola Soldani ${ }^{1}$
}

Resumo

La catástrofe es la dimensione ontológica que permite un desarrollo de nuevas prácticas políticas ligadas a los eventos naturales.

Los ámbitos se cruzan y se aprovechan los eventos naturales para la creación de orden políticos, que aunque relacionado con la tradición política occidental cobran nuevos significados y producen relaciones de poder.

En la esfera jurídica Agamben habla de estado de excepción, en aquella medica Foucault traza la frontera entre medicalización y política y finalmente Bataille une religión y filosófia ponendo el milagro en el centro de la soberanía. En la dialéctica entre seguridad y catástrofe la sociologia del riesgo de Beck y Luhmann es una clave impescindible para interpretar los eventos.

¿El repensamiento de la solidaridad será una salida plausible para estos tiempos?

\section{Del Estado de excepción como dispositivo y del dispositivo como concepto}

¿Qué es el estado de excepción hoy en día? Es muy difícil responder de manera directa y completa a esta pregunta. Creo que el estado de excepción es algo cuya definición trasciende el ámbito estrictamente jurídico del estado de sitio. Su sentido moderno tiene origen en la democracia de la Francia revolucionaria, en el Siglo XVIII. El jurista argentino Gabino Ziulu, autor de una

\footnotetext{
${ }^{1}$ Doctorando de la Facultad de Ciencias Sociales, Universidad de Buenos Aires, becario del CONICET y del Insitituto de Investigación Gino Germani (IIGG) Buenos Aires.
} 
monografía sobre este mismo argumento, ya en el prólogo de dicha obra nos restituye la complejidad del tema afirmando que "el estado de sitio es (...) una de las instituciones previstas en la Constitución argentina que más ha sido subvertida y desnaturalizada en su funcionamiento" (Ziulu, 2000 p. VII). Pero como decía antes, a pesar de la complejidad en el ámbito constitucional, el sintagma "estado de excepción" más allá de ser un terminus tecnicus del derecho, empieza en la última década a tener un significado filosófico más significativo.

Quien traslada el concepto a la filosofía con aptitud genealógica es Giorgio Agamben, que en el año 2003 publica el libro Stato d'eccezione (Agamben, 2004) ${ }^{2}$, en el cual toma el concepto foucaultiano de dispositivo para definir el estado de excepción:

El termino dispositivo nombra lo que se realiza en una pura actividad de gobierno (oikonomia) sin algún fundamento en el ser (Agamben, 2007). El gobierno es un arte práctico, una manera de hacer que forma parte de la capacidad inventiva y de la fuerza circulante de las técnicas: de acá la consecuencia que el concepto de gobierno no puede ser autosuficiente porque se inscribe en un orden instrumental, se revela solo en la prestaciones que brinda (Napoli, 1997, p.11).

Así en el ámbito del derecho pasa que el estado de excepción:

(...) señala un umbral en el cual lógica y praxis se indeterminan y una pura violencia sin logos pretende actuar un enunciado sin ningún referente real... en el cual la potencia y el acto son separados radicalmente, es ciertamente algo así como un elemento místico o, sobre todo, una fictio, a través de la cual el derecho busca anexarse la propia anomia (Agamben, 2004 p.81-3).

Es en esta fictio donde el problema de la norma y de su aplicación, que no está de ninguna manera contenida en ella (la aplicación en la norma) ni puede ser de ella deducida, se explica la esencia del estado de excepción excepción, Adriana Hidalgo editora, Buenos Aires, 2004, cit. p. 83 y 81. 
Soldani, Giulio Nicola. Políticas de la catástrofe. Nuevo paradigmas entre excepción, gobierno, riesgo y soberania.

donde a través de una "actividad práctica", que "no es en algún modo lógica", norma y aplicación de la norma se manifiestan en su paradójica relación, produciendo lo normal.

Es decir, que la norma se aplica a la realidad des-aplicándose, suspendiéndose para aplicarse ${ }^{3}$.

Pero ¿por qué tanto interés en esta forma de reflexión filosófica aparentemente introducida en el contexto filosófico político del Siglo XX por Carl Schmitt?

Para responder a esta pregunta, tenemos que delinear un pequeño excursus conceptual que recorra algunas etapas importantes con el fin de comprender más a fondo los aspectos fundamentales de este dispositivo.

Una primera respuesta (múltiple) nos la ofrece Jacob Taubes, quien en una conferencia de 1985 cuenta su primera experiencia con el texto más importante de Schmitt sobre el estado de excepción, el libro Politische Theologie (1922). La Teología política le parecía fundamental porque a través de sus formulaciones "era un ataque (...) a la modernidad liberal, tanto como una forma de vida, como también una forma de ciencia: una crítica del espíritu moderno". El autor marca la importancia del concepto de guerra civil permanente, propuesto por Schmitt también en otras obras, respecto de un dispositivo como el estado de excepción, que sanciona el fin de la posibilidad de normativizar las relaciones bélicas o conflictivas. Taubes demuestra particular interés, al ser profesor de filosofía, en la relación de estas temáticas con la historia, ya que "sin historia no hay verificación".

También para Foucault el papel de la historia en relación con la filosofía es algo crucial para entender la naturaleza y el funcionamiento de los dispositivos. No se puede entender la importancia de su contribución al pensamiento político contemporáneo sin analizar los diferentes aspectos de este hecho. Para nosotros, es importante hoy el carácter histórico de su argumentación sobre la posibilidad histórica de las epistemes "desde grado de aceptabilidad histórica hasta el sistema de aceptación (Foucault, 1997 p.56)", es decir, una práctica "arqueológica" y de las formas de ontología del presente.

\footnotetext{
3 "Todas las instituciones sociales se han formado a través de un proceso de desemantización y de suspensión de la praxis concreta en su inmediata referencia a la realidad" (Agamben, 2004 p. 50)
} 
La pregunta sobre la actualidad “¿Qué es el presente que estamos viviendo?” es complementaria al problema “¿Cómo no ser excesivamente gobernados?"

Por fin, Walter Benjamin habla de un estado de excepción como tradición de los oprimidos, cuyo desarrollo bajo una perspectiva revolucionaria podría crear la posibilidad de una liberación del derecho y subvertir la teoría schmittiana de la decisión soberana sobre el estado de excepción, abriendo a una posibilidad nueva, en la cual "la humanidad podrá jugar con el derecho como hacen los niños con los objetos fuera de uso (...) liberándolos de su uso canónico". Entonces, en el marco de una teoría de la soberanía, el estado de excepción no se caracteriza solo por su peculiar aspecto espacial y topográfico dentro del derecho (Agamben), sino también por su relación con el tiempo, por lo que casi se podría hablar con Benjamin de una "secularización del tiempo en espacio".

En Homo Sacer (Agamben, 1995) Agamben había dedicado los primeros capítulos de dicha obra a las relaciones entre soberanía y estado de excepción, criticando al mismo jurista nazi, por el contrario en Stato

d'eccezione (Agamben, 2003) se encuentra un desarrollo de esas ideas, cuyo núcleo es la interpretación del supuesto debate esotérico entre Carl Schmitt y Walter Benjamin sobre el Ausnahmezustand.

El estado de excepción es aquel dispositivo puente entre derecho y anomia, que abre a las tecnologías gubernamentales de nuestro mundo liberal (y neoliberal) y que, en cuanto dispositivo, encuentra su eficacia en la historia que el mismo produce.

\section{Seguridad: especialización del poder entre normalización disciplinaria y prácticas gubernamentales}

En más de una ocasión Foucault describió la gestión de enfermedades tomando como ejemplo la diferencia entre la modalidad de gestión de la lepra, por un lado, y la de la peste, por el otro. 
Soldani, Giulio Nicola. Políticas de la catástrofe. Nuevo paradigmas entre excepción, gobierno, riesgo y soberania.

La gestión de esta segunda enfermedad es el ejemplo de una nueva actitud del poder: un poder que se renueva y que empieza, en lugar de reprimir y prohibir, a normalizar y producir:

...sueño político de la peste, en el cual el poder político se ejercita plenamente (...) la peste es el momento en el cual la subdivisión exhaustiva de una población es llevada a su punto extremo, cuyas ramificaciones capilares llegan sin interrupción a la grana de los individuos mismos (Foucault, 2000 p.50).

Es evidente como las técnicas de control sobre la peste implican una nueva utilización del espacio (a la vez urbano y como categoría). "Los médicos son los inventores de los espacios" decía Foucault, y así explicaba la importancia de la reflexión clínica para el nacimiento y el desarrollo de las practicas de seguridad y también sus maneras de inscribirse en el espacio. Nos enfrentamos a dos factores (el médico y el espacial) que abren una nueva dimensión en la cual la vida humana depende cada vez más de la adecuada construcción del medio ambiente. Este, como nueva configuración del espacio en relación al viviente, es uno de los fines de las acciones del gobierno, su manera indirecta de intervención sobre la vida garantizándola, alimentándola, regulándola, controlándola, asegurándola y gestionándola.

Lo que evidencia una gran diferencia en el campo de la medicina es la introducción de la vacuna contra la viruela (que sigue al tratamiento de la peste), "el problema no es la exclusión como en el caso de la lepra, ni de la cuarentena como en el caso de la peste, sino de la epidemia".

La seguridad, por su parte, se apoyará en una serie de datos materiales (y no en la separación y en los espacios vacíos como la disciplina)... no se trata de que la seguridad reconstruya ese dato de tal manera que sea dable esperar un punto de perfección, en este sentido se aprovecha de elementos que ya existen. Por lo tanto, se trabaja no sólo sobre datos naturales sino también sobre cantidades que son relativamente reducibles, pero nunca por completo. Como jamás se las puede anular, se trabajará sobre probabilidades... Se trata simplemente de maximizar los elementos positivos y minimizar, al contrario, los aspectos riesgosos e inconvenientes... y se abrirá en cambio hacia un porvenir 
no exactamente controlado ni controlable, no exactamente medido ni mensurable; el buen ordenamiento de la ciudad será justamente eso: tener en cuenta lo que puede pasar. En síntesis, creo que se puede hablar de una técnica que en lo fundamental se ajusta al problema de la seguridad, es decir, en el fondo, al problema de la serie. No será concebida ni definida de los elementos que se desplazan ... Serie indefinida de acontecimientos que se producen ... etc. Lo que caracteriza en esencia al mecanismo de seguridad es, creo, la gestión de esas series abiertas y que, por consiguiente, sólo pueden controlarse mediante un cálculo de probabilidades (Foucault, 2005 p. 16).

En estas frases Foucault se refiere a la evolución de la seguridad urbana, pero es evidente cómo se podría aplicar todo esto a la gestión de la epidemia. Esto significa que la seguridad implica una nueva relación de carácter inmediato entre salud y espacio, en la cual la población es el sujeto y el ambiente el objeto. "El espacio propio de la seguridad remite entonces a una serie de acontecimientos posibles, remite a lo temporal y lo aleatorio, una temporalidad y una aleatoriedad que habrá que inscribir en un espacio dado" (Foucault, 2005). Además "el espacio en el cual se despliegan series de elementos aleatorios es, me parece, más o menos lo que llamamos un medio. El medio es, por supuesto, una noción que en biología recién aparece -como bien sabrán- con Lamarck" (Foucault, 2005 p. 16).

En La cittá biopolitica Andrea Cavalletti nos explica, mediante un análisis de las ciencias policiales alemanas y de la policía médica italiana del siglo XVIII, cómo a pesar de la relación directa entre ambiente y población surge el elemento económico.

La población que corresponde al dispositivo de seguridad implica de allí su justa distribución, siempre es dotada de un carácter espacial inmanente. Todas la teorías "económicas" sobre la proporción de los medios de subsistencia pueden ejercitarse a partir de esta economía política o espacialización primaria...Hay un espacio fundamental de la población -entre lo normal y lo patológico (Canguilhem)- al cual el crecimiento de las fuerzas ... deberá ser conmensurada. $\mathrm{Y}$ es operando en referencia a esta primera espacialización 
Soldani, Giulio Nicola. Políticas de la catástrofe. Nuevo paradigmas entre excepción, gobierno, riesgo y soberania.

que la policía médica inducirá sobre la misma población sus efectos benéficos. (Cavalletti, 2005)

Antes de terminar esta primera parte genealógica y pasar a analizar aspectos ya tematizados, que le pertenecen a hechos históricos más recientes, como el terremoto en Italia el año pasado (2009), hace falta aclarar que "no hay sucesión ley-disciplina-seguridad, sino que la seguridad es una determinada manera de añadir y hacer funcionar, además de sus propios mecanismos, también las antiguas armaduras de la ley y de la disciplina"(Foucault 2005) y que la pregunta que orientará nuestra mirada hacia dichos eventos será la cuestión de la relación entre los dispositivos de seguridad y el estado de excepción, que no solo le pertenecen a las catástrofes sino también y sobre todo a nuestra realidad cotidiana y a nuestra identidad porque:

los dispositivos tienen siempre que implicar un proceso de subjetivación, es decir, tienen que producir su sujeto (Agamben, 2006).

\section{6 de Abril del 2009}

Cuando a las 3:32 de la madrugada del 6 de abril del 2009 la tierra temblaba en la ultra centenaria ciudad apenina de L'Aquila, en el centro de Italia, según las escuchas de la policía ya había empresarios hablando por teléfono y alegrándose por el gran negocio que se podría lograr de esta situación de emergencia.

Dicho terremoto causó la muerte de 309 personas (Italia es un país muy sísmico, en el siglo pasado murieron más de 160.000 personas a causa de terremotos). La gestión del estado de emergencia y también la reconstrucción de la ciudad fue encomendada al Dipartimento di protezione civile.

En el año 1992 la Presidencia del Consejo de los Ministros de Italia, que corresponde al gabinete del jefe del gobierno (Italia tiene un sistema parlamentario y no presidencial), fundó // Dipartimento di Protezione civile (Ley 
225/92), una entidad cuya tarea es la actividad de prevención de catástrofes naturales, defensa de la población civil y de gestión de las situaciones excepcionales.

En el 2001, con un decreto, el presidente reformó la protección civil trasladándola bajo directo control de él mismo y del ministro del interior como su delegado.

Las tareas que la ley le impone son de "Pronóstico - Prevención Socorro - Recuperación" pero queda, sin duda, y no claramente expresada, la de gestionar el caso excepcional.

A través de este nuevo órgano el gobierno logró durante una década completa gestionar diferentes situaciones escapando a cada control.

La gestión de esta emergencia en el caso de L'Aquila es paradigmática. Por un lado, hubo un aparato mediático dentro de la Protezione Civile que logró producir la imagen de la gestión perfecta ${ }^{4}$, por el otro, hubo un intento de prolongar la situación de emergencia para aprovecharla en lugar de solucionar el problema lo más rápido posible.

Una masiva utilización del ejército y de empresas privadas de seguridad fue lo que los ciudadanos incrédulos tuvieron que sufrir. La aplicación de medidas de supuesta seguridad, tanto extremas como inútiles, la imposición rígida de conductas dentro de los campos de evacuados (en carpas), fueron solo una parte de la prolongada violencia que los aquilanos llegaron a conocer.

En efecto, la construcción de campos, cerrados por la supuesta inseguridad, como alojamiento temporáneo es una de las características de esta nueva gestión y es radicalmente diferente de lo ocurrido en otras ocasiones (terremoto Umbria-Marche 1997), en las que se prefirió utilizar vivienda-contenedores, formando barrios abiertos. Dentro de los campos los desplazados estaban sometidos al albedrío de los "capo-campo" elegidos por la Protección civil bajo ningún criterio legal. Estos seres humanos mantenidos en vida y privados de cualquier actividad más que la supervivencia misma nos hacen recordar mucho a figuras humanas, nude-vite, ya descritas por

\footnotetext{
${ }^{4}$ Un sobreviviente se expresó así un mes después de la catástrofe "quello che il Governo sta facendo sulla loro pelle è un gigantesco banco di prova per vedere come si fa a tenere prigioniera l'intera popolazione di una città, senza che al di fuori possa trapelare niente", en una carta a su mujer, publicada después en la pagina web

http://www.informa-azione.info/abruzzo ho visto laquila lettera a mia moglie scritta ieri notte.
} 
Soldani, Giulio Nicola. Políticas de la catástrofe. Nuevo paradigmas entre excepción, gobierno, riesgo y soberania.

Agamben, pero también al mismo tiempo a todos aquellos inmigrantes encerrados en Italia, a unos kilómetros de todo esto, en los Centros de Identificación y ( $\mathrm{CIE})$, verdaderas excepciones permanentes.

De los cuatros aspectos que componen cada catástrofe (prevencióndestrucción-socorro-reconstrucción), para la población el tercero fue el más insoportable, ya que el tiempo que llevó la gestión de la excepción les permitió producir una reflexión crítica, y sus necesidades artificialmente prolongadas y por eso amplificadas, los empujaron hacia la organización de diferentes acciones directas, desde la remoción de los escombros del centro de la ciudad, cerrado por la policía, hasta una última propuesta de ley de iniciativa popular ${ }^{5}$ que se presentará en ocasión de la marcha del 20 de noviembre del $2010{ }^{6}$

La entidad que sigue gestionando la formación de los campos y regulando la vida dentro de estos, se llama Di.coma.c (Dipartimento comando e controllo). Cuanto se ha dicho antes sobre la diferencia entre la gestión de la lepra, de la peste y de la viruela, puede ahora volverse útil para analizar estos hechos.

La peste es el momento en el cual la subdivisión exhaustiva de una población es llevada a su punto extremo, decía Foucault, y la disciplina es el medio para conseguir este nivel de control.

Son los médicos los inventores de los espacios políticos -también una cita de Foucault- y no es casualidad que Guido Bertolaso (el jefe absoluto de la Protezione Civile), con su mentalidad "del hacer", sea un exponente de esa categoría profesional, acostumbrado a las emergencias sanitarias sobre todo en África y Asia en contextos (post-)coloniales, donde el respeto a las reglas tampoco era imaginable. ${ }^{7}$

Más allá de la gestión de la emergencia y de sus peculiaridades hay otro aspecto fundamental,

\footnotetext{
${ }^{5}$ En el ordenamiento jurídico italiano el referendum y la legge di iniziativa popolare son definidos como elementos de democracia directa.

${ }^{6}$ El texto de la propuesta de ley puede leerse en italiano en la página http://www.carta.org/articoli/19867. Para llegar a ser debatida en el parlamento, la propuesta necesita 50.000 firmas de ciudadanos y ciudadanas que la apoyen.

${ }^{7}$ No es causalidad que el mismo día en que se presentó el proyecto de ley popular para la reconstrucción de la ciudad (7-11-2010), Guido Bertolaso dejó su puesto en la Protezione Civile. Casi parece que la historia haya querido demonstrar que la tarea del manipulador puede terminar solo cuando la mayoria de los humanos accionan.
} 
la Protezione Civile representa en Italia una nueva modalidad de "ser fuente de derecho" no obstante lo dispuesto. Inaugura una nueva modalidad de gobernar. Se puede afirmar que crea una realidad jurídica paralela dentro de la cual vive y de la que se alimenta.

Es casi como enfrentarse con un metadispostivo, que califica el carácter de los eventos (y así de la realidad), y declara la excepcionalidad de los eventos, más allá de una declaración oficial del estado de sitio o también de la efectiva excepcionalidad del hecho mismo. De esta manera, se vive constantemente en una situación de potencial emergencia que solo depende de la decisión soberana, siendo esta una manera de justificar la intervención de la Protezione Civile.

En Italia parece cada vez más evidente lo que hace siete años ha escrito Giorgio Agamben

Esto significa que el principio democrático de la división de los poderes hoy se ha devaluado y que el poder ejecutivo ha absorbido de hecho, al menos en parte, al poder legislativo. El parlamento no es más el órgano soberano al cual corresponde el derecho exclusivo de obligar a los ciudadanos a través de la ley; se limita a ratificar los decretos emanado del poder ejecutivo. En sentido técnico, la República no es más parlamentaria sino gubernamental (Agamben, 2004 p.51-2).

A pesar de esta situación, se desarrolla una nueva tecnología de gobierno. En el caso particular de la Protezione civile, se produce un nuevo nivel de intervención, un nuevo campo de acción que se posiciona al lado de la emergencia sin llegar a su gravedad: el grande evento.

Un grande evento es aquel evento que, sin tener ningún elemento de excepcionalidad ni tampoco la declaración del estado de sitio relativa al evento en sí, se entrega a la gestión legibus soluta de la protección civil.

En el párrafo 5 del artículo 5bis de la reforma del 2001 se dice claramente que los grandi eventi son, en materia de normativa, equivalentes a las calamidades. 
Soldani, Giulio Nicola. Políticas de la catástrofe. Nuevo paradigmas entre excepción, gobierno, riesgo y soberania.

En tiempos de paz, la protección civil tiene su tarea seguridaria de defensa de la vida humana y del control permanente del territorio a través de obras de prevención y estudios sobre el territorio.

Es en tiempos de guerra o de catástrofe natural o calamidad que la protección civil puede obrar con ordenanza en derogación a la disposición vigente (pero respetando los principios generales del ordenamiento jurídico y la constitución), en estos casos el jefe de gobierno tiene que declarar el estado de sitio, indicando claramente los límites espaciales y temporales de dicho estado en relación con la naturaleza de los eventos. De esta manera se produce un mecanismo parecido a la dictadura comisaria propuesta en el ámbito constitucional por Carl Schmitt en su libro de 1921, Die Diktatur.

Es decir, que no se puede tolerar la apertura de un estado de semianomia y de casi absoluta discrecionalidad de un comisario no electo (cuyas actividades están libres de control de la Corte Constitucional -Suprema - y de la Corte dei conti - la Corte de control del presupuesto nacional -) sin delimitar sus términos de espacio y tiempo.

Pero ¿cómo puede justificarse la emisión de un decreto de excepcionalidad de un evento si el contexto no lo permite?

En marzo del 2003 el gobierno (con un decreto, más tarde transformado en ley) declaró el estado de sitio. La motivación era la emergencia internacional relacionada con la guerra de Irak, cuya coparticipación se declaró en esos días. Al mismo tiempo se decidió entregar, siempre por medio de una ordenanza, los poderes excepcionales para la emergencia relacionada con la crisis internacional al comisario delegado, jefe de la Protección civil (Guido Bertolaso).

De esta manera, se empezó una actividad de gobierno paralela y frenética, libre de controles sobre la legalidad de la utilización de sus recursos (gastos y presupuestos). En ocho años y medio se han emitido 587 ordenanzas con un gasto publico, lejos de cualquier tipo de transparencia, de 10600 millones de euros (Bonaccorsi, 2009 p. 10).

Volviendo a la pregunta inicial, ¿qué son los grandi eventi, entonces?

La definición que se dio en el ámbito del debate parlamentario fue 
... cualquier evento agravado de una excesiva multitud de personas, con el factor simbólico que juega, requiere la activación de medidas adecuadas de previsión y de prevención del riesgo y de fortalecimiento, en clave sinérgica, de las fuerzas para utilizar en ocasión de posible emergencia (Bonaccorsi, 2009 p. 14).

Es decir, cualquier evento social podría ser un grande evento, pero si se analiza en detalle, la mayoría de las ordenanzas se refieren a eventos que requieren fuertes inversiones de capitales para la realización de obras públicas.

De esta manera, se financia sin control a las empresas privadas con dinero público y se produce un cortocircuito político. No solo se produce una confusión entre las situaciones excepcionales y las no excepcionales (y que hablando en términos de derecho positivo y de estado de derecho, era lo que señalaba Agamben) sino que se abre una zona de indistinción entre derecho y economía, totalmente discrecional, que afecta la gestión supuestamente democrática de las instituciones locales.

El decreto que regula los grandi eventi los pone, jurídicamente hablando, al mismo nivel que las catástrofes, libres de controles de presupuesto y de gasto, como eventos excepcionales.

De hecho, esta estructura excepcional llega a una profundidad tal en el ordenamiento jurídico del país, que produce un desencuentro entre actividades económicas y jurídicas en el financiamiento de algunas estructuras públicas. Perpetrando las condiciones excepcionales hace también un uso totalmente arbitrario del dinero público y de las estructuras democráticas.

Pero lo que más nos interesa es el poder que tiene la Protección civil de emitir ordenanzas, poder que le sirve para realizar sus eventos (y que puede llegar hasta el orden de desalojo de un campo de gitanos, por ejemplo) y que revela su faceta más autoritaria.

La institución nacida para enfrentar la calamidad deviene una calamidad para el ordenamiento jurídico italiano. La verdadera emergencia parece ser la democrática (Bonaccorsi, 2009 p.6). 
Soldani, Giulio Nicola. Políticas de la catástrofe. Nuevo paradigmas entre excepción, gobierno, riesgo y soberania.

\section{Risikogesellschaft}

A mediados de la década de los ochenta el sociólogo alemán Ulrich Beck inauguró el debate sobre la Sociedad de Riesgo (Risikogesellschaft), con la publicación del libro Risk Society: Towards a New Modernity (La sociedad de riesgo: hacia una nueva modernidad). Después de unos años apareció en forma de respuesta parcial Soziologie des Risikos (Sociología del Riesgo) por Niklas Luhmann y finalmente en el año 2002 de nuevo por Ulrich Beck, Weltrisikogesellschaft (La sociedad mundial de riesgo).

Lo que más nos interesa en este debate son los binomios riesgo y catástrofe, riesgo y decisión, y la relación entre ellos.

La tesis originaria de Beck es que el riesgo ha penetrado los ámbitos sociales, el trabajo con la flexibilización laboral, la producción de energía con accidentes atómicos, etc., y que sea parte de una nueva dialéctica de la modernidad, que conlleva la constante producción de paradojas. La característica principal de la Risikogesellschaft es la pérdida efectiva de su centro gravitacional, que es la seguridad, y, al mismo tiempo, la incesante búsqueda de esta como ficción espectacular (en el sentido debordiano de separación radical de la realidad de sus representaciones).

Para comprender mejor este tipo de sociedad hay que reflexionar sobre algunos aspectos que le pertenecen al riesgo, que son constitutivos de su ser y de su funcionamiento. Se podría decir que el riesgo también es un metadispositivo, así como lo son el estado de excepción, la catástrofe y el espectáculo, es decir, un dispositivo que tiene la potencialidad de activar una gran cantidad de dispositivos, que además dependen de su funcionamiento.

El riesgo es una anticipación de la catástrofe... y, al revés,...la catástrofe es la trasformación del riesgo en realidad (Beck, 2007). Dentro de la esfera de la realidad de la catástrofe, el riesgo es la posibilidad de prever y calcular las consecuencias de los hechos catastróficos futuros.

Tanto Beck como Luhmann individúan el nacimiento conceptual de la actitud social frente al riesgo con la invención de Pascal, en el siglo XVII, del cálculo de probabilidades y con su difusión.

Según su significado etimológico, catástrofe es el vuelco del orden de las cosas, del orden de los eventos. Poder calcular las efectivas posibilidades de 
cambio y el grado de incertidumbre de estos, fue lo que le ofreció la reflexión matemático-filosófica a la teoría liberal.

En su afirmarse, el liberalismo conjugaba la realización de un proyecto económico-político ligado a la expropiación-apropiación-producción-venta con la necesidad de una atenuación de la posibilidad de fracaso que el cálculo de las probabilidades parecía garantizarle.

El cálculo del riesgo es parte de una maquinaria histórica que parte de su condición, es decir, que sigue aferrada demasiado tiempo a los riesgos que fueron determinados o rechazados ... aquello que queda como resto no resuelto, a pesar de todos los esfuerzos antagonistas por una realización ordenada, es lo que llamamos riesgo (Luhmann, 1991 p.35) .

Así Luhmann define el riesgo y a pesar de esta definición el sociólogo alemán introduce una distinción fundamental entre riesgo (Risiko) y peligro (Gefahr).

Entre estos dos términos hay un medio que es el daño: siempre hay un daño como consecuencia de una catástrofe, siempre el daño es la configuración de algo futuro en el presente inmediato.

El daño se configura, entonces, como peligro cuando su origen se atribuye al medio ambiente. El riesgo, diversamente, es algo que se produce a partir de una decisión.

Es con este fundamental ámbito decisorio que necesitamos cruzar el discurso anterior sobre la relación temporal entre riesgo y catástrofe, ya que el riesgo hace el futuro decidible en el presente (Beck, 2007).

Como en el pensamiento de Carl Schmitt, la decisión se pone en el centro de la producción de sentido de la realidad. Lo que asombra es encontrar de nuevo dicha categoría trasladada del derecho público al estudio de la sociedad occidental neoliberal. La analogía no termina aquí, tomar el riesgo significa ponerse en juego, es la secularización de la confesión, dice Luhmann, un programa de reducción al mínimo del arrepentimiento.

En este sentido se desarrolla un nuevo concepto de seguridad vinculado de manera inequívoca a la decisión. Aquí está quizá la diferencia entre Schmitt y los análisis de Beck y Luhmann, no se trata más de una decisión soberana, 
Soldani, Giulio Nicola. Políticas de la catástrofe. Nuevo paradigmas entre excepción, gobierno, riesgo y soberania.

sino de una decisión vinculada al cálculo. Este vínculo suyo le confiere una fuerza más, porque tiene también una legitimidad económica, que calcula la relación entre daños y recursos, que calcula su potencial biopolítico.

La seguridad es solo seguridad para la decisión que realmente tiene en cuenta el riesgo y la decisión sobre

... el concepto de seguridad -que- sigue siendo un concepto vacío... funge como un concepto de reflexión o también como concepto válvula-deescape... que sucede cuando el contexto que guía la evaluación del riesgo constituye el mismo otro riesgo (Luhmann, 1991 p. 70)?

En la sociedad de riesgo no existe la posibilidad de un afuera de la decisión, en una hipertrofia de la decisión, no decidir también significa elegir.

La evaluación del riesgo es lo más importante, porque es algo que no puede interrumpirse en ningún momento, los riesgos son continuos y es en cada momento que hay que elegir, en este sentido la decisión sobre el riesgo es constitutiva de esta sociedad: la prevención solo puede realizarse a pesar de decisiones continuas para elegir, entre los muchos, cuál riesgo tomarse.

Es en la posibilidad de transformar el presente en futuro (intentando garantizar la sobrevivencia a las catástrofes) que la decisión sobre el riesgo ejerce su potencialidad y, sin embargo, en esta economía de gobierno y de previsión vale la paradoja enunciada por Luhmann: los riesgos constituyen peligros y los peligros riesgos.

A pesar de esta enunciación y de su posibilidad sobre el tiempo, se manifiesta el carácter milagroso de la decisión para el riesgo, esta aparece como algo que se impone desde arriba y que impregna la realidad.

Exactamente el carácter de milagro junto a la paradoja produce una extraña dimensión, que

Comparé au terme de risque, celui de catastrophe dramatise l'extension et l'intensité des boulevermentes, le degré de interrupción possible du cours normal des événements; alors que semble-t-il, paradoxalement, rien ne change, et rien en s'interrompt vraiment... Notre hypothese, c'est que cette banalisation 
de la dramatisasion des événements es une de composents fundamentales de la biopolitique contemporaine (Neyrat, 2008 p.12).

Lo que quiere decir Neyrat es que cada vez más se pone el riesgo y la amenaza dentro de situaciones que no presentan una eventualidad tan extrema. Sin embargo, en previsión o luego de una catástrofe algo a veces pasa. Si se trata de una catástrofe natural, o de una guerra, se olvidan pronto las victimas y se agradece al destino, optimizando la fuerza destructiva de la catástrofe para lograr algo como un nuevo New Deal local. En este sentido, para la economía ha pasado algo milagroso en el significado latino de cosas prodigiosas. Naomi Klein no ha descubierto nada nuevo, hablando de la "Doctrina Shock", aunque sin duda se trata de un trabajo de investigación periodística fundamental, en 1958 Hannah Arendt escribía

puede ser conveniente reflexionar sobre el llamado "milagro económico" de la Alemania de postguerra, milagro solamente si se le considera en un anticuado marco de referencia. El ejemplo alemán muestra claramente que bajo condiciones modernas la expropiación del pueblo, la destrucción de objetos y la devastación de ciudades pasan a ser radical estimulante para un proceso no de simple recuperación, sino de más rápida y eficaz producción de riqueza ... en Alemania, la destrucción completa de ciudades ocupó el lugar del implacable proceso de todas las cosas mundanas, que es la marca de la economía del derroche en la que vivimos ... bajo las condiciones modernas, conservación, significa ruina (Arendt, 2003 p.282).

Si el escenario postbélico de hace 50 años era así, hoy han cambiado muchas cosas, como la gestión por empresas privadas de las ayudas humanitarias, o la misma conducción de la guerra, sin duda la categoría de riesgo gana cada vez más importancia

Devorados por la hiperinflación, y demasiado endeudados como para negarse a las exigencias que vienen de la mano de los préstamos extranjeros, los gobiernos - de los países afectados por catástrofes- aceptaban el "tratamiento de choque", creyendo en la promesa que los salvaron de mayores desastres (Klein, 2007 p.32). 
Soldani, Giulio Nicola. Políticas de la catástrofe. Nuevo paradigmas entre excepción, gobierno, riesgo y soberania.

\section{Decisión-Milagro-Soberanía}

En la ambigüedad que vincula el riesgo a la catástrofe y pone la decisión come elemento determinante, está la peculiaridad de esta "segunda modernidad". La espera de un hecho catastrófico, hasta su misma producción artificial (a través la banalisation de la dramatisasión) adquiere cada vez más el carácter de una esperanza, no solo en su sentido común, sino también en el sentido más teológico de las tres virtudes teologales: Fe, Esperanza y Caridad. Esta segunda abraza un ámbito bastante grande, que tiene como extremos la expectación confiada en las gracias de Dios y el miedo de provocar su castigo.

En esta condición de espera, en la cual se ponen los humanos, se evidencia la "deslimitación del estado de excepción" (Beck, 2007 p.118). Nuevamente fuera del ámbito estrictamente jurídico, Beck evoca la actualización agambeniana de las tesis de Benjamin y de Schmitt sobre el Ausnahmezustand.

Volvemos rápidamente a los años veinte y retomamos una muy conocida expresión schmittiana

"El estado excepcional tiene en la Jurisprudencia análoga significación que el milagro en la teología"(Schmitt, 1922).

Pero hay una modificación de la noción de milagro propuesta por Schmitt. Para él

el deísmo, con una teología y una metafísica que destierran del mundo el milagro y no admiten la violación con carácter excepcional de las leyes naturales implícitas en el concepto de milagro y producido por intervención directa, como tampoco admiten la intervención directa del soberano en el orden jurídico vigente. El racionalismo de la época de la ilustración no admite el caso excepcional en ninguna de sus formas (Schmitt, 1922).

Hay, entonces, una relación directa entre milagro y soberanía, sobre todo en relación al poder del soberano de producir un quiebre dentro de las normas constitutivas del cuerpo jurídico, del estado de derecho. Pero milagroso 
no es solo el poder del soberano, sino también su esencia misma. Es decir, el milagro es al mismo tiempo constitutivo de la posición del soberano, de la posición soberana (libre de cada vínculo superior excepto aquello divino) y de su poder libre de las leyes. Su posición legitima y permite su poder, su poder consolida su posición.

La asociación entre milagro y soberanía, como parte de una substancia divina en la tierra -aquí se ve el teísmo schmittiano- genera, a través de la decisión, el estado de excepción.

Este es un primer aspecto de la posible relación entre soberanía y milagro.

Otra perspectiva sobre el tema ha sido propuesta por Bataille, "que también permanece como un pensador de la soberanía" (Agamben, 1995). Al igual que Schmitt también para Bataille cada realidad tiene un carácter religioso, pero para él cada forma religiosa tiene carácter soberano. La unidad fundamental es definida por Bataille como sentido de la soberanía, como "esfuerzo milenario del hombre a la búsqueda de un lugar donde convergieron todas las suertes milagrosas de este mundo" (Bataille, 1996).

La soberanía es milagrosa porque es la soberanía de la excepción. La excepción reside esta vez en el hecho de que el soberano goza de las prerrogativas que la mayoría de los humanos, que en relación a su soberanía solo son objetos, añoran.

En Bataille sin embargo lo que limita la soberanía no es el ejercicio de la norma, sino el cálculo vinculado a la producción, y al mismo tiempo, es la soberanía que tiene el poder de romper el círculo de la producción y del trabajo. Esto porque en Bataille la "soberanía tiene poco que ver con la de los Estados... sino que... pertenece esencialmente a todos los hombres que poseen y nunca han perdido del todo el valor atribuido a los dioses y a los 'dignatarios' " (Bataille, 1996 p. 63).

La pluralidad de la referencia divina y su manifiesta pertenencia a una multiplicidad de sujetos es lo que distingue la soberanía de Bataille de la de Schmitt. Los dos autores se refieren a dioses diferentes para formular su concepto de soberanía. También las finalidades son bien distintas, la soberanía de Schmitt tiene como fin establecer un orden, que implica al mismo tiempo la designación del enemigo y la trasgresión del orden mismo para su 
Soldani, Giulio Nicola. Políticas de la catástrofe. Nuevo paradigmas entre excepción, gobierno, riesgo y soberania.

reafirmación. Es una operación de objetivación de una condición determinada que necesita designar el componente extraño que hay que combatir, porque con su existencia amenaza el orden. El Estado es la única unidad de poder que tiene la misma legitimidad que tiene el Dios único del catolicismo, que se concreta en una relación temporal, que interrumpe la circularidad cronológica del mundo pagano (y quizá natural).

La soberanía del Estado es su capacidad de lograr el orden mediante el carácter trascendente-divino de la decisión.

En Bataille el elemento temporal se da de esta manera, "no vemos en el horizonte el momento soberano, en el que no cuente nada más que el momento mismo, en efecto, aquello que es soberano es disfrutar el tiempo presente sin mirar a otra cosa que no sea el tiempo presente"(Bataille, 1996).

De manera opuesta a Schmitt, la soberanía está lejos del ejercicio del poder. Su elemento en común es más bien la posibilidad de trasgredir el orden, pero si para Schmitt la trasgresión es un elemento fundamental de autoridad, divinamente milagroso, para Bataille trasgredir la prohibición, acerca lo humano a lo animal y pone los personajes que encarnan la soberanía, reyes y curas, en una zona de indistinción entre dignidad humana y dignidad soberana. De hecho, estas personas quedan encerradas en una dimensión suspendida entre prohibición y trasgresión, en la cual las dos cosas conviven, el rey divino, en cuanto ser humano, puede gozar su soberanía solo a pesar de la limitación que el ejercicio de esta le impone. Se crea en este sentido un contraste entre soberanía y poder, entre soberano y sociedad. Con respecto a la sociedad, él se incorpora continuamente aunque sea para ella peligro mortal y bien supremo.

El alcanzar a tocar al mismo tiempo la vida biológica de cada uno y la muerte social es tal vez la característica más esotérica y más importante del concepto de la soberanía de Bataille. Su extensión entre estos dos extremos sintetiza la función que ella tiene y su misma esencia, siempre móvil, nunca definida de una vez por todas.

En dicha extensión aparece claramente el elemento determinante de la incalculabilidad que articula de manera inequívoca la soberanía con el milagro que es un fenómeno extraño a la lógica del cálculo y que permite vivir a pleno el momento presente, superando las dicotomías morales entre útil e inútil, 
bueno y malo. Milagro y soberanía son constitutivos el uno del otro, rompiendo la estructura binaria del humano. Se abre una dimensión nueva, que lejos de ser sintética, desarticula articulando una diferente percepción del orden: junto con el milagro irrumpe la NADA.

tenía conciencia del carácter banal de esas relaciones, pero terminó por hacer cegadora una luz que me deslumbraba. Una pequeña frase de Goethe sobre la muerte: «Una imposibilidad que de pronto se hace realidad», tuvo el mérito, sin pretenderlo, de hacer sensible a mis ojos el carácter milagroso del acontecimiento más temido. Pero lo más sorprendente era la identidad de reacciones no calculadas, que hacía, desde un punto de vista definido, desaparecer la diferencia entre lo positivo y lo negativo, la extrema felicidad y la desdicha extrema, situando lo uno y lo otro, indiferentemente, en el punto de resolución de nuestros pasos. Lo más claro era que, esencialmente, un impulso irracional daba el valor soberano al milagro, aunque éste fuera desdichado (Bataille, 1996 p. 76).

Si Bataille tiene razón se puede ver cómo la reconfiguración del orden en la NADA atribuye al milagro aquella característica que los modernos atribuyen a las catástrofes: la capacidad de renovar y de anular para recomenzar. La diferencia profunda está en el hecho de que el milagro contribuirá a una soberanía sin cálculos que se acerca paradójicamente a la pura calculabilidad de la situación catastrófica. En el medio de las dos, la vida humana será cada vez más zoé y cada vez menos bios, muy cerca de la animalidad en el primer caso y solo una vida biológica, que tiene que ser mantenida en vida en la segunda.

En una nota al pié Bataille escribe

Sin embargo, es desde hace mucho tiempo que no aparece nada que se pueda juzgar como nuevo, la tormenta es más fuerte, las olas son más grandes, la última se calmó sólo después de un grado de intensidad tan alto que, gracias a una semi llamarada que ilumina todas las cosas, parecen surgir perspectivas ya conocidas. Pero son las perspectivas de la razón que excluyen el elemento de imprevisibilidad, imposible, que yo llamo un milagro. Los trastornos causados por las nuevas técnicas (muy diferentes de las de las 
Soldani, Giulio Nicola. Políticas de la catástrofe. Nuevo paradigmas entre excepción, gobierno, riesgo y soberania.

revoluciones sociales que actúan en el marco de la ley) nos sorprenden en la medida en que siguen viviendo en la espera de cambios milagrosos. Pero no revelan nada más profundo que la permanencia de esta espera... No hay nada comparable a los efectos que llevaron al trabajo, cuyos efectos conjuntos pusieron fin al equilibrio secular y dieron a luz a una producción catastrófica (Bataille, 1976).

Con esta cita podemos retomar las tres ideas sobre catástrofe-milagroriesgo que Neyrat, Arendt y Klein nos sugirieron.

La de la banalisation de la dramatisasión de la catástrofe, para que todo cambie sin cambiar nada, que se combina con la tesis de Arendt sobre la destrucción como impulso productivo. Se destruye para volver a producir, para consumir, en una supuestamente eterna cadena de destrucción y reconstrucción.

\section{Para no concluir}

Los hechos de nuestra actualidad nos ofrecen muchas posibilidades de reflexionar sobre las múltiples relaciones entre seguridad, estado de excepción, gestiones disciplinarias y soberanía.

No es nada completamente nuevo. Tanto la teoría política como la reflexión filosófica del siglo $\mathrm{XX}$, y en parte también del siglo $\mathrm{XIX}$, han ofrecido muchos argumentos a este debate, que parecen concretarse cada vez más.

"Las tendencias históricas de nuestros tiempos afirman o temen... el triunfo de un estado de excepción que deviene la norma, según un modelo de afirmación de Normale Katastrophen" (Ortmann, 2003 p.88) nuestra mirada se cruza con aquella del ángel de la historia de Walter Benjamin que mirando al progreso, (para nosotros, el de las tecnologías de gobierno) ve "una única catástrofe que amontona incesantemente escombros sobre escombros (Eine 
einzige Katastrophe, die unablässig Trümmer auf Trümmer häuft)", o a la casa de Juan Salvo en Vicente López, donde en el marco de la historieta El Eternauta (Oesterheld, Solano Lopez, 2004 p.39), pocos humanos se encuentran mirando los copos de nieve asesinos caer y matar en una trama de macabra tanatopolítica.

Luhmann en la obra antes citada afirma que el mecanismo de las ayudas institucionalizadas, y también el de las ayudas humanitarias, rompe cada forma de solidaridad social frente a la catástrofe

La reciprocidad no puede activarse sin una disposición a la compensación, mientras que la utilización de las ayudas organizadas no obliga a nada.

Entre las características de las ayudas organizadas está la de generar ningún tipo de solidaridad... El futuro es producido en forma de futura decisiones, y de fragilidad organizada. Hay instancias decisionales y hay afectados (Luhmann, 1992 p. 151).

Klein muestra, veinte años más tarde, que el mecanismo de las ayudas se ha transformado en negocio, no solamente desapareció la solidaridad, sino también este ámbito se transformó en el ámbito de la más explícita competencia, mecanismo que intercambia con la vida el interés económico, la guerra como nuevo paradigma económico político, la sacertas de la política de los derechos humanos, que mata sin sacrificar, da la muerte sin buscar más ningún discurso de legitimidad.

Si la desaparición constante del límite entre excepción y norma hace de la catástrofe un paradigma necesario para el mantenimiento en vida del 
Soldani, Giulio Nicola. Políticas de la catástrofe. Nuevo paradigmas entre excepción, gobierno, riesgo y soberania.

dispositivo económico-político de nuestros tiempos, solo la amistad política puede ejercer como contradispositivo

... La amistad de la cual el placer es inseparable por qué no es "externa a nuestras necesidades". Porque, si no se debe desear la seguridad, se puede sin embargo encontrarla en la amistad, que no se reduce a la utilidad: "no necesitamos tanto las ayudas de los amigos - dice Epicuro- cuanto la confianza en sus ayudas". Ahora, la actualidad de la amistad o de la liberación epicúrea está para Fallot en la solidaridad revolucionaria (Cavalletti, 2009, p.92-3).

\section{Bibliografia}

Agamben, G. Che cos'è un dispositivo? 2006. Roma : Nottetempo, 2006.

Agamben, G. Homo Sacer. II potere sovrano e la nuda vita. 1995. Torino : Einaudi, 1995.

Agamben, G. Stato d'eccezione. Homo Sacer II, I 2003. Torino : Bollati e Boringhieri, 2003.

Arendt,H. La condición humana. 2003 Buenos Aires, Barcelona, Mexico : Paidos, 2003.

Bataille, G. Lo que entiendo por soberanía.1996. Barcelona : Paidos, 1996.

Beck, U. La socied del riesgo global. 2008. Buenos Aires, Barcelona Mexico : Paidos, 2008.

Benjamin, W. Sul concetto di storia. 1997. Torino : Einaudi, 1997.

Bonaccorsi, M. Potere assoluto. La protezione civile ai tempi di Bertolaso. 2009. Roma :

Edizioni Alegre 2009.

Cavalletti, A. Classe. 2009. Torino : Bollati e Boringhieri, 2009.

Cavalletti, A. La città Biopolitica. 2005. Milano : Bruno Mondadori, 2005.

Foucault, M. Illuminismo e critica. 1997. Roma : Donzelli, 1997.

Foucault, M. Los anormales. 2000. Mexico, Argentina, Brasil, Colombia, Chile, España, Estados Unidos de America, Perù, Venezuela : Fondo de cultura económica, 2000.

Foucault, M. Seguridad territorio población. 2006. Mexico, Argentina, Brasil, Colombia, Chile, España, Estados Unidos de America, Perù, Venezuela : Fondo de cultura económica, 2006.

Luhmann, N. Sociologia del Riesgo. 1992. Mexico : Universidad Iberoamericana, 1992.

Napoli, P. Il governo e la critica. 1997. Roma : Donzelli, 1997.

Neyrat, F. Biopolitique des Catastrophes. 2008. Paris : Editions MF, 2008.

Oesterheld - Solano Lopez, El Eternauta. 2004. Buenos Aires : Biblioteca de la historieta Clarín, 2004.

Ortmann, P. Regel und Ausnahme. Paradoxen soziales Ordnung. 2003. Frankfurt am Main : Suhrkamp, 2003. 
Pulifiato, A. Protezione civile SPA. Quando la gestionde dell'emergenza si fa business. 2010. Roma : Alberti Editore, 2010.

Taubes, J. In divergente accordo. Scritti su Carl Schmitt, 1996. Macerata : Quodlibet, 1996.

Ziulu, G. A., Estado de sitio. ¿Emergencia Necesaria o autoritarismo en encubierto? 2000. Buenos Aires : Depalma 2000. 